\title{
Le fragment dans l'autoportrait littéraire : entre éclatement et unité
}

\author{
Johanne Lalonde \\ Université du Québec à Trois-Rivières
}

Définir « les formes brèves » en littérature fait appel à un ensemble de méthodologies traitant du phénomène de la brièveté. Car comment distinguer les proverbe, maxime, fable, nouvelle, épigramme, aphorisme, slogan et fragment, dont la brièveté varie sensiblement? Non seulement il y a variation de longueur d'une forme brève à une autre, mais la notion de brièveté possède ses propres nuances au sein de chacune d'elles. Sans compter qu'elles varient selon les époques et leur niveau de réception. Par exemple, à la fin du XIXe siècle, Oscar Wilde avait inventé une panoplie d’aphorismes, généralement prisés par un auditoire mondain et bourgeois ${ }^{1}$. Mais aujourd'hui, à l'ère de la diversité culturelle, joyau de la

\footnotetext{
${ }^{1}$ L'aphorisme est une phrase dont la teneur réfère à un principe moral ou à un théorème quasi-scientifique. Exemple d'aphorisme inventé par Oscar Wilde : « Le drame de la vieillesse, ce n'est pas que l'on se fait vieux, c'est qu'on reste jeune » (n.p.).
} 
mondialisation, les formules aphoristiques à saveur déclamatoire suscitent un doute à l'égard des abus que pourraient contenir leurs généralisations. Et que dire de la popularité des slogans revendicateurs lors des conflits politiques? Des raccourcis idéologiques dans la propagande? Toutes ces questions relèvent autant de la sociolinguistique que de l'analyse du discours. Plus précisément, elles convoquent l'interdisciplinarité des sciences du langage.

En ce qui a trait à l’art du bref en littérature, le terme qui attire mon attention est celui de « fragment », car les formes littéraires dites « brèves » ont en commun la concision de leurs énoncés sauf, pour l'écriture fragmentaire qui indépendamment de sa longueur ou de sa brièveté, suggère une interruption au cœur de l'inachevé. C'est une abréviation (déviation) dans la doxa de la représentation dont les principes s'appuient sur le caractère achevé et unitaire d'une œuvre. Alors pourquoi s'attarder sur le fragment? Car ce n'est pas tant sa longueur qui le caractérise, il peut s'avérer court ou long, tout dépend de la forme que son auteur cherche à créer ou à déconstruire. En partie, c'est lorsque le processus d'une œuvre est abrégé, laissant sa création « en chantier », que le fragment se rallie à la brièveté. Le fragment rompt avec la synthèse d'un tout unifié, ce dernier demeurant indéfinissable, voire indéterminé ou pratiquement inexistant. Or, l'écriture fragmentaire pourrait se définir mais pas seulement, par une absence de rapport avec un ensemble plus large. Et si l'œuvre s'appuie sur un recueil de fragments rassemblés autour d'un axe conceptuel, chaque fragment se suffira à lui-même sous l'autarcie de sa propre topique.

Soulignons que j'aborde le fragment en tant que création littéraire, et non pas en tant que document archéologique abimé par le temps ou en tant que manuscrit non finalisé. C'est l'auteur qui sur le plan de sa pratique, adopte une posture d'écriture en concluant un pacte avec l'infinitude ou la non-finitude de son œuvre. Tandis que les règles du classicisme le reléguaient aux rebuts sans aucune considération, le fragment est devenu monnaie courante 
dans les pratiques contemporaines de l'écriture ${ }^{2}$. Surtout en ce qui a trait à l'autoportrait

littéraire, car ce genre fait référence à un espace de représentation fragmenté par la mise en abyme de «l'écrivain écrivant», qui du coup, se distancie de la narration propre à l'autobiographie pour glisser vers les louvoiements vagabonds d'une écriture introspective³. L'acte narratif est un dispositif d'écriture indispensable à rendre compte du cours des événements, tandis que le fragment offre un lieu de représentation où le temps s'arrête sur une phénoménologie d'existence.

\section{Le fragment : genre littéraire ou pratique d'écriture?}

Dans son essai portant sur L'écriture fragmentaire. Définitions et enjeux, Françoise SusiniAnastopoulos (1997) établit une différence fondamentale entre la figure scellée de l'aphorisme et la forme ouverte du fragment. Le premier s'appuie sur le caractère concis de la forme dont la figure de style est une saisie claire et définitive de l'énoncé. Alors que le deuxième suggère l'idée d'un inachèvement, d'une écriture en discontinu et dont les formes côtoient les abimes de la déconstruction plutôt que le circuit linéaire d’un récit ou d'une intrigue. Il est aussi question du problème que pose la dialectique fragment-système, à savoir dans quels paramètres se situe le texte fragmentaire en tant que genre ou sous-genre. Sur ce point, je résiste à la consécration générique du fragment, car si ce dernier reste à la fois l'essence de la poésie, du journal intime, du roman épistolaire et plus, il se situerait plutôt au

2 Sur la théorisation du fragment sous sa forme «aphoristique » et de ses enjeux, génériques, esthétiques et gnoséologiques, voir Susini-Anastopoulos (1997). À ce sujet, deux exemples méritent d'être mentionnés. Bien que poétesse, romancière, essayiste et dramaturge, la canadienne Nicole Brossard présente un répertoire d'œuvres dont les enjeux s'appuient sur une pratique d'écriture essentiellement fragmentaire. Pour sa biographie et son œuvre, voir sa fiche technique sur le site de l'Académie des lettres du Québec, http://www.academiedeslettresduquebec.ca/membres/nicole-brossard-39. Du côté des Français, les œuvres du romancier, critique littéraire et philosophe Maurice Blanchot, relèvent non seulement de cette pratique, on pense tout de suite à L'écriture du désastre (1980), Le pas au-delà (1973) et L'entretien infini (1969) par exemple, mais l'auteur offre également un répertoire d'essais sur le fragment dont entre autres, L'espace littéraire (1988 [1955]).

3 Afin de nuancer cette opposition binaire entre narration et autoportrait, il convient de mentionner L'image fantôme, d'Hervé Guibert (1981); un autoportrait en fragments composé de petits récits. 
niveau d'une pratique d'écriture apte à transcender tous les genres. Il se pourrait même qu'il traverse un répertoire, qu'importe l'éventail des genres empruntés par l'artiste.

Selon Susini-Anastopoulos, la pratique du fragment serait marquée par un intérêt grandissant en faveur d'une esthétique qui cherche à rompre avec un certain nombre de maniérismes issus du classicisme et ce, depuis le tout début du XvII siècle. Bien que son ouvrage reste centré sur le texte fragmentaire aphoristique, l'auteure marque un point dans l'histoire du fragment : «Pour que l'on s'intéresse à ce peu, cet encore ou désormais presque rien, il a fallu que se produise une véritable inversion des catégories esthétiques, donnant peu à peu naissance au "phénomène fragmentaire" » (Susini-Anastopoulos, 1997, p. 2-3). Non seulement s'est-il instauré en tant que résultat légitime de lecture au fil des siècles, mais de son procès, nait son propre cogito. Et en quoi l'écriture fragmentaire aurait-elle contribué au caractère autoportraitiste de certains textes tels que Roland Barthes par Roland Barthes (Barthes, 1975), Autoportrait (Levé, 2013 [2005]) ou La ballade du calame (Rahimi, 2015)?

Dans une volonté de circonscrire l'autoportrait littéraire par rapport à l'autobiographie, Philippe Lejeune (1975) et Michel Beaujour (1980) s'entendent sur une notion de rupture entre l'autoportrait et le chronologique. Le fragment permettant un espace de mise en représentation indépendante et autocratique, offre ce lieu d'écriture contrenarratif. Quant à sa représentation fragmentaire, l'autoportrait évoque la cristallisation d'une personne au temps présent avec ses passé et futur soudés presque sans distinction. Dans le même ordre d'idée, Beaujour insiste sur le fait que la rhétorique de l'autoportrait « se fonde à la fois sur un autre rapport à l'écriture, et sur une spatialisation qui déploie aussi bien l'intérieur que l'antérieur dans une extériorité topique.» (p.349). Voilà qui appelle à la contiguïté des figures dans l'autoportrait littéraire. Et paradoxalement, en dépit d'une impossible autoreprésentation du sujet dans sa totalité, l'autoportraitiste, dans sa quête 
d'unité, se retrouve devant l'exigence de créer une ou des figures. Le fragment, au lieu d'être un système ouvert sur l'infini, se referme sur une topique allant de la métaphore au récit bref.

Les illustrations qui suivent portent sur le fragment en tant que pratique d'écriture dans l'autoportrait littéraire. Tout d'abord, je le présente comme étant un traitement formel capable d'atteindre à l'unité d'une œuvre avec Autoportrait, de Levé (2013 [2005]). Ensuite, il sera question de l'absence de totalité dans l'autoreprésentation de Roland Barthes par Roland Barthes (1975). Tandis que Levé atteint l’unité de son œuvre au moyen d'énoncés éclatés, Barthes renonce à la totalité avec une figure d'autoportrait désarticulée. Je conclurai donc sur la poésie des figures inachevées d’Atiq Rahimi dans La ballade du calame (2015).

\section{Édouard Levé et l'unité fragmentaire}

Levé était un artiste interdisciplinaire d'origine française; il a été peintre, photographe et écrivain. Le thème du suicide est récurrent dans ses œuvres ${ }^{4}$, tel un projet se dévoilant avec parcimonie et qui a malheureusement abouti en octobre 2007. Concernant son œuvre Autoportrait, publiée deux ans auparavant, l'auteur respecte le pacte autobiographique, c'està-dire qu'il s'engage à révéler des traits de sa personne réelle dans une forme littéraire que l'on nomme l'autoportrait. Le pacte autobiographique consiste en ce que l'œuvre présente des faits biographiques ressortissant de la vie de son auteur (Lejeune, 1975, p. 13-46). Et dans le cas d'un autoportrait, le fait biographique se transpose à un trait de sa personnalité sociale versus les jardins secrets de sa personne, au symbole incarné d'un souvenir, à un artefact, une

\footnotetext{
${ }^{4}$ Voir du même auteur, Suicide (2008); une œuvre posthume qui évoque et interroge le suicide d'un ami cher avant que l'auteur ne commette son propre suicide le 15 octobre 2007, à l'âge de 42 ans. Selon Paul OtchakovskyLaurens, ancien directeur littéraire chez P.O.L. Éditeur, Édouard Levé avait déposé son manuscrit dix jours avant de se suicider, soit le 5 octobre 2017.

Voir également sur le site de P.O.L. Éditeur, trois articles de journaux portant sur cet ouvrage, http://www.polediteur.com/index.php?spec=livre\&ISBN=978-2-84682-236-7.
} 
habitude particulière, une légende marquante, etc. Voici un premier exemple d'une écriture engagée dans ce pacte autobiographique et qui prend forme dans l'autoportrait de Levé :

En train, dans le sens opposé à la marche, je ne vois pas les choses arriver, mais partir. Je ne prépare pas ma retraite. J'estime que la meilleure partie d'une chaussette est le trou. Je suis inattentif à la quantité d'argent sur mon compte en banque. Mon compte en banque est rarement dans le rouge. Shoah, Numéro Zéro, Mobutu roi du Zaïre, Urgences, Titicut Follies et La Conquête de Clichy m'ont plus marqué que les meilleures fictions. (Levé, 2013, p. 9)

Les démarcations entre autobiographie et autoportrait sont loin d'être franches, car le fait biographique tangue entre une mémoire sélective et la prétendue stagnation de ses oublis. Si bien que d'une œuvre à l'autre, de nombreux glissements peuvent se produire; l'autobiographie présentera des digressions vers un discours s'approchant davantage d'une rhétorique de figures et l'autoportrait instrumentalisera la narration, mais tous deux s'appuieront sur la biographie de son auteur, que ce soit sous forme narrative ou fragmentaire. Donc, pour établir une distinction opératoire, je suggère que l'autobiographie est une condition d'écriture qui relève d'un acte narratif relié au passé, parce que l'auteur raconte une ou plusieurs de ses histoires en empruntant un modèle temporel linéaire propre au récit. Et quant à l'autoportrait, il est un acte poétique centré sur le présent, car il s'approprie un espace de figuration par la fragmentation de l'être et des topiques qui le représentent ${ }^{5}$. L'acte figuratif ou l'acte poétique doivent être pris au sens d'une topique qui

\footnotetext{
${ }^{5}$ Pour une typologie primaire des genres autobiographiques, j'attribue respectivement, un acte littéraire, ainsi qu'une direction temporelle aux trois formes principales que sont l'autobiographie, l'autoportrait et l'autofiction (autobiographie/acte narratif du passé; autoportrait/acte poétique du présent; autofiction/acte performatif du futur). Selon le corpus analysé, les catégories s'inverseront, comme dans l'autobiographie présentialiste du journal intime ou l'autofiction partant d'un événement passé par exemple. J'insiste donc sur la nature primaire de cette typologie qui en principe, devra être secondée par une autre, de même que par une troisième et ce, non seulement en fonction de la temporalité de l'œuvre, mais aussi, selon l'acte littéraire choisi par l'écrivain. Car rien n'interdit de raconter son autobiographie sous forme de poésie ou par le biais d'une partition de fragments. Pas plus que l'autoportrait ne s'appuiera exclusivement sur la poésie ou sur les figures de styles pour dépeindre son auteur(e). Nonobstant, ce sont les axes littéraires de l'œuvre qui selon moi, différencient les genres, soit par l'événement (temps), soit par l'être (espace), ou par le devenir (performatif qui se joue entre temps et espace).
} 
crée un espace de représentation isolé, et où la forme brève possède son système sémantique autonome ${ }^{6}$.

Sans vouloir m'engager davantage dans des débats que ces notions pourraient soulever, l'autoportrait d'Édouard Levé restitue cette idée de figuration à caractère présentialiste. Autoportrait est entièrement construit sur un déroulement de texte sans paragraphe, ni chapitre, juxtaposant une phrase à la suite de l'autre et dont les énoncés très brefs demeurent pour la plupart, arbitraires les uns par rapport aux autres. Chaque phrase est une pièce de mosaïque qui elle, révèle différents pans de l'être. Le tout s'enchaîne dans un mouvement de circonvolution jusqu'à la fin du livre. La composition du texte crée donc l'effet d'une série photographique désordonnée, telle une pile de photos prises au hasard :

J'ai parlé à Salvador Dali à l'âge de deux ans. La compétition ne me stimule pas. Décrire précisément ma vie me prendrait plus de temps que la vivre. Je me demande si, en vieillissant, je deviendrai réactionnaire. Assis jambes nues sur du skaï [cuirette], ma peau ne glisse pas, elle crisse. J'ai trompé deux femmes, je leur ai dit, l'une y fut indifférente, l'autre pas. Je plaisante avec la mort. Je ne m'aime pas. Je ne me déteste pas. Je n'oublie pas d'oublier. Je ne crois pas que Satan existe. Mon casier judiciaire est vierge. J'aimerais que les saisons durent une semaine. (Levé, 2013, p. 7)

Certaines phrases relèvent de l'autobiographie comme les rapports d'infidélité. Rien n'est raconté en détail, mais ce sont des actions qui présentent un ordre de conséquences. D’autres énoncés restent centrés sur les caractéristiques de l'individu, et ces dernières ne sont aucunement expliquées dans une logique de chronologie d'événements, mais plutôt décrites comme un système d'idiosyncrasies tirées du néant. La représentation fragmentée permet de passer par toutes sortes de postures délibérées pour ensuite bifurquer vers des traits cognitifs accidentels, génétiques, inexplicables, mais bien réels.

\footnotetext{
${ }^{6}$ Sur la « Fonction poétique », voir Roman Jakobson (1963), Essais de linguistique générale, Paris, Minuit, chapitre 11; «Linguistique et poétique », p. 209-248. Et sur l'autarcie absolue du « Mot» ou du « Nom », voir également Roland Barthes (1972 [1953]), Le degré zéro de l'écriture, Paris, Seuil, p. 35-42.
} 
Ce type d'énonciation peut paraître simpliste au premier abord, mais l'œuvre présente une plus grande complexité en dépit de la banalité apparente du discours. Le tissu du texte alterne sensiblement d'énoncés aux phrases courtes à ceux dont les phrases sont beaucoup plus longues. Et parfois, les énoncés s'acoquinent en groupe autour d'une thématique :

Je fume rarement plus de dix cigarettes par jour, ma gorge a une jauge naturelle qui fait qu'au-delà je suis écœuré. Il m'arrive de ne pas fumer pendant plusieurs jours. J'ai arrêté de fumer plusieurs fois par accident, toujours de la même manière : à l'occasion d'une angine, je ne fume plus et, guéri, j'oublie de reprendre. Je fume des cigarettes roulées car elles se consument à la vitesse à laquelle je tire dessus, si elles s'éteignent, je les rallume, les cigarettes toutes faites se consument seules et m'imposent un rythme dont je ne veux pas être tributaire. (Levé, 2013, p. 43)

De la phrase unique aux groupuscules à thèmes, le rythme de lecture suit les modulations de l'être à la manière d'un graphique et produit une saisie tangible de la personne qui se décrit. En ce sens, le cumul des fragments consolide la figure d'autoportrait, exactement à l'image de la page couverture créée par l'auteur qui était aussi dessinateur7. Levé a utilisé une technique pointilliste pour réaliser son autoportrait dont une moitié disparaît complètement dans le noir. Cette image néo-impressionniste de l'auteur reste représentative de la posture littéraire de l'œuvre qu'est l'inépuisable fragmentation de l'être en petites unités.

Dans cet amalgame de traits personnels, parfois communs à plusieurs d'entre nous, Autoportrait possède son fil d'Ariane menant à des digressions narratives où l'auteur raconte des anecdotes. La narration est instrumentalisée dans le but de représenter un souvenir fétiche, un événement stocké dans le sanctuaire de sa mémoire. Tel un objet muséal, le fait biographique reste à l'abri de l'oubli. Ainsi truffée d'anecdotes, l'œuvre alimente la lecture d’une vive curiosité, sans compter l'effet comique que suscite la prose de Levé :

Mon père m'a surpris en train de faire l'amour avec une femme, lorsqu'il a toqué à la porte, j'ai dit mécaniquement: "Entrez», son visage s'est illuminé, il a aussitôt

${ }^{7}$ Couverture du livre Autoportrait, dessin de Levé, http://urlz.fr/6jk2. 
refermé la porte, lorsque l'amie a tenté de repartir discrètement, il s'est précipité vers elle et lui a dit : « Revenez quand vous voudrez, mademoiselle. » (Levé, 2013, p. 15)

D'autres extraits relatent des faits biographiques appartenant aux personnes de son entourage et qui ont marqué Levé, comme cet oncle à l'humour intrépide par exemple :

Un de mes oncles avait le goût du scandale et du jeu, il volait dans les magasins juste pour rire, il achetait Hara Kiri et me le faisait lire, il faisait semblant d'être handicapé mental sur la plage, sautait en hurlant et en bavant sur une femme qui bronzait, il posait des questions avec des mots qui n'existent pas à une fermière voisine, il faisait croire au téléphone à des inconnus qu'un serpent les attendait à Orly, il jouait au casino jusqu'à s'en faire volontairement et définitivement interdire, il tentait de récupérer le loyer des boîtes de nuit que son père avait gagnées au poker et finissait saoulé par les locataires mafieux qui l'amadouaient au champagne. Je ne joue pas au casino. (Levé, 2013, p. 13)

C'est avec cette dernière phrase que Levé clos la séquence narrative tout en affinant son autoportrait, parce qu'il se définit lui-même par rapport à son oncle et ce, par une négative. L'auteur résout ici ce rapport ambigu entre le réel de l'autre tissé dans les fondations du Moi (anamnèse du suicide et Hara Kiri) et la frontière qui les différencie nettement l'un de l'autre. L'autoreprésentation passe ainsi par les figures d'altérité ayant laissé des palimpsestes chez l'auteur. En outre, les récits avec ses oncles donnent à voir des variations qui vont de la personnalité cocasse aux circonstances dramatiques d'une rencontre ou d'un décès :

Un de mes oncles est mort du sida peu après la faillite de la galerie d'art dans laquelle il avait tout investi. Un de mes oncles a rencontré l'homme de sa vie en conduisant lentement sa voiture rouge décapotable dans les rues de Paris, l'homme en question, un immigrant hongrois, était désespéré, et marchait au hasard avant de se suicider, mon oncle s'est arrêté à sa hauteur et lui a demandé où il allait, ils ne se sont plus quittés jusqu'à ce que la mort les sépare. L'ami de mon oncle m'a appris à rire de ce que je voyais à la télévision, et qui n'avait, à priori, rien de drôle, par exemple la coiffure de Bobby Ewing dans Dallas. Je n'ai pas signé de manifeste. (Levé, 2013, p. 15)

À bien observer les trois citations qui précèdent, force est de constater que la phrase unique en tant que fragment autonome et possédant toute l'information nécessaire à chaque énoncé et sans contredit, Levé manie les mots avec une économie remarquable - constitue l'élément 
fondateur de l'œuvre. Cette structuration de l'autoportrait par la juxtaposition de phrases indépendantes est un procédé littéraire propice à la sérialisation des figures d'auteur dans l'autoportrait. Non seulement l'unité fragmentaire de l'œuvre passe par un balayage quasitopographique des plans physique, émotionnel, cognitif, sexuel et existentiel de l'auteur, mais c'est précisément sa sociabilité qui alimente les anecdotes et qui active l'extension systématique des phrasés. Car lorsque Levé raconte ces récits très brefs, il inclut dans une seule et longue phrase, l'incipit, le développement et l'excipit. Chaque action est séparée par une virgule et le point n'arrive qu'à la fin de l'historiette.

Il convient également de souligner qu'il sépare les personnes par des phrases distinctes : l'oncle mort du sida, la rencontre amoureuse d'un deuxième oncle, de même qu'il termine avec une autre phrase pour indiquer ce que l'ami de l'oncle lui a appris. En ce sens, il est à noter que la citation précédente à ces trois dernières et qui traite de sa préférence pour les cigarettes roulées, sectionne les caractéristiques de cette habitude à fumer en ponctuant chaque information distincte d'un point final. Levé applique des règles de ponctuation qui marquent les différents territoires de son autoreprésentation. La syntaxe étant construite sur des modèles récurrents, donne du relief au texte en créant des espaces différenciés entre ce qui appartient à l'excentricité de l'individu (chaque phrase correspondant à un point unique dans l'autoportrait), et les bulles narratives où la mémoire se raconte et où des profils de personnalités ayant imprégné la vie de l'auteur se dessinent (la virgule marquant les temps d'action d'un événement ou d'une circonstance à l'intérieur d'une même phrase). Édouard Levé a ainsi réalisé un autoportrait pointilliste au moyen de deux procédés littéraires : la phrase-fragment et la ponctuation distinguant le caractère individuel de ses récits fétichisés. 


\section{Roland Barthes et l'impossible totalité}

L'autoportraitisme est un exercice éminemment fragmentaire. Dans la peinture par exemple, le portrait est étudié et reproduit à partir d'un seul angle avec une expression unique, celle-ci étant maintenue artificiellement pour que soit réalisable la cohésion de l'œuvre, ou du moins, pour que lui soit restituée un peu de ressemblance avec la personne qui se peint elle-même. Et même si les traits sont rendus de façon parfaitement réaliste, l'autoportrait ne représente qu'une partie de l'être réel. Car comment rendre une image complète par la photo ou le portrait peint, si l'angle choisi est de profil ou au trois-quarts? Nous ne voyons que cette perspective figée dans le temps où «l'avant» et «l'après » disparaissent instantanément. Et bien sûr, il y a les fragments du visage dont la physionomie se sépare en plans d'ombre et de lumière, en traits et en courbes. Ce sont des lieux de fragmentations se tenant au milieu de ce fragment d'être qu'est l'autoportrait dans son ensemble. En effet, l'autoportrait littéraire possède cette multiplicité de plans et de perspectives que comporte non pas le visage comme tel, mais plutôt des états d'être interloqués ou disloqués.

Dans Roland Barthes par Roland Barthes, l'auteur commentant une photo de lui, assis sur la pelouse en compagnie de sa mère, inscrit ce court texte, tout juste au-dessus de la photographie : «D’où vient donc cet air-là? La Nature? le Code? » (p. 38). Son expression contraste avec l'air radieux de sa mère. Il semble résilient et le fragment accompagnateur met de l'avant la topique d'une question dualiste communiquant à la fois, l'intérieur et l'antérieur du sujet. Le texte interloque la confusion actuelle de l'écrivain face à une image de lui-même : ce témoin d'un passé qu'il a du mal à saisir. Quelle part de son être avait-il révélée ou fabriquée dans ce cliché? Non seulement l'autoreprésentation correspond à un moment furtif de sa vie, à une fraction d'être, mais elle reste indécise quant à la nature ou au code de son expression faciale. La figure demeure ouverte sur une dialectique. Le souvenir est fragmenté 
par une question. Contrairement à la page 29, où une autre photographie suit un court texte sur l'ennui et montre une image de l'écrivain avec une expression franche. Le titre en appuie l'énoncé : «Ennui : la table ronde». L'ambivalence et le doute ont disparu, le temps d'un accablement.

Suite à la série photographique qui revient constamment sur l'indécidabilité de l'auteur à se saisir et à se définir, Barthes entreprend de se représenter sur le plan exclusivement sémantique, c'est-à-dire sous la forme d'une encyclopédie. Les termes choisis suivent un ordre alphabétique, mais chacun s'annonce comme étant arbitraire les uns par rapport aux autres et de ce fait, le tout demeurera non-exhaustif. L'autoportrait ou la figure énonciative demeure difficilement saisissable, car l'écrivain porte au-devant de son discours, le structuralisme dont il est profondément tributaire. Le structuraliste s'efface derrière un consort de considérations sans vraiment réussir à décrire le sujet parlant. Les fragments restent en état d'ouverture où tous les éclats sont permis. La figure énonciative de l'œuvre demeurera désarticulée ne sachant réduire l’opacité entre le penseur et l'être pensé8.

L'extrait qui semble toutefois correspondre de plus près à sa figure d'écrivain est « Le cercle des fragments » (Barthes, 1975, p. 96-98). L'auteur étant un adepte assidu de l'écriture fragmentaire, se met en représentation entouré des fragments ayant marqué sa vie littéraire :

Écrire par fragments : les fragments sont alors des pierres sur le pourtour du cercle : je m'étale en rond : tout mon petit univers en miettes; au centre, quoi? Son premier texte ou à peu près (1942) est fait de fragments; ce choix est alors justifié à la manière gidienne «parce que l'incohérence est préférable à l'ordre qui déforme » [citation d'André Gide]. Depuis, en fait, il [Barthes] n'a cessé de pratiquer l'écriture courte : tableautins des Mythologies et de L'Empire des signes, articles et préfaces des Essais critiques, lexies de S/Z, paragraphes titrés du Michelet, fragment du Sade II et du Plaisir du Texte. (Barthes, 1975, p. 96-97)

\footnotetext{
${ }_{8}$ Dans une étude plus approfondie de l'autoreprésentation chez Roland Barthes, son œuvre posthume Journal de deuil (2009) mériterait d'être analysée sous l'angle de l'altérité. Contrairement à Roland Barthes par Roland Barthes (1975), un autoportrait soutenu par un regard solipsiste, le journal portant sur le deuil de sa mère réalise un autoportrait fragmentaire à travers l'expérience de l'autre, de la figure maternelle au cœur du Moi. Voir également Adam Watt (2014) qui, dans une perspective intertextuelle de l'autobiographie, repère l'écriture de Proust dans l'œuvre de Barthes.
} 
Le fragment en lui-même se fait topique de l'autoreprésentation du sujet, car il fait partie de ses pratiques d'écriture ayant donné naissance à son répertoire d'œuvres. D'autant plus que ces dernières ont été reconnues pour leur importante contribution à la littérature française. Si le fragment fait figure d'autoportrait dans les premiers paragraphes, le texte se transforme rapidement en un discours d'argumentation sur la topique. L'extrait qui suit permet de différencier la rhétorique de figure de la citation précédente et son glissement vers l'essai : «Le fragment a son idéal : une haute condensation, non de pensée, ou de sagesse, ou de vérité (comme dans la Maxime), mais de musique : au "développement", s'opposerait le “ton", quelque chose d'articulé et de chanté, une diction: là devrait régner le timbre.» (Barthes, 1975, p. 98). L'auteur ancre sa figure d'autoportrait dans le répertoire de ses écrits fragmentaires pour enfin disserter sur l'essence du fragment.

S'il lui paraît illusoire d'atteindre à une forme qui serait totalisante de lui-même, Barthes insiste sur le fait que l'écriture par fragments demeure semblable aux cycles d'une pièce musicale, parce que chaque fragment préserve son autonomie tout en disposant d'une souplesse de cohabitation avec les autres partitions. Il y aurait des interstices de voisinage où chaque topique est conviée au rassemblement d'une topique-mère que je choisis de nommer « la figure énonciative de l'œuvre ». Par exemple, lorsqu'il écrit Mythologies (1957), les objets littéraires propres à chaque fragment cohabitent sans aucune parenté entre eux, mais tous se réunissent sous la même bannière d'analyse : dépister à la lumière de la pensée structuraliste, le construit idéologique des produits culturels de son époque (nourriture : « Le vin et le lait », « Le Bifteck et les frites »; sport: « Le Catch» [« La Lutte» pour le Québec]; et les fabulations excessives de la consommation bourgeoise avec entre autres, "Saponides et détergents », etc.). 
Quant à son autoportrait Roland Barthes par Roland Barthes, le carrousel encyclopédique est une somme de fragments qui aboutit au Monstre de la totalitép; un court texte suivi d'un dessin (p. 182-183) démontrant l'inconfort de l'auteur à s'autoreprésenter. La figure de cette chute incarne un humanoïde fait de linéaments branchus. C'est une rhétorique texte-image qui évoque précisément le discours de l'auteur sur le fait autoportraitiste en concluant sur son impossible totalité. Toute tentative d'y parvenir ne réussirait qu'à produire une forme monstrueuse. Tandis que Levé atteint l'unité par l'autarcie de chaque phrase en tant que fragment, le thème de la totalité chez Barthes produit une figure ébréchée, car l'œuvre ne possède pas la figure énonciative d'une topique-mère où l'autoportrait serait composé autour d'un seul axe conceptuel. Des photos à l'encyclopédie, les textes sillonnent sans but, chaque fragment demeurant ouvert sur une forme incomplète ou à venir, sur une pensée à naître.

Le Monstre de la totalité avec sa silhouette hideuse et sans peau, semble plus unifié que l'ensemble des textes. La figure fermée du monstre ne représente en rien le caractère inachevé et désarticulé des fragments qui l’ont précédée. Roland Barthes reste centré sur cet irrésistible attrait pour les débuts qui deviennent des rebuts sans finalité. La pensée discursive et aléatoire prime sur la mise en forme d'une autoreprésentation par figures de style. C'est pourquoi Barthes privilégiait le journal de bord où tout est possible et à partir duquel il engrosse, biffe, laisse tel quel, améliore, déconstruit ses fragments, sans souci d'atteindre au stade de l'achèvement. Puisant sa matière première dans le journal de bord, le fragment serait pour Barthes, l'art de l'inachevé, une forme abrégée où l'incomplétude devient la figure même de son autoreprésentation. Roland Barthes par Roland Barthes, présentant un assemblage de fragments qui oscille entre le discours intérieur et les topiques extérieures,

\footnotetext{
9 Sur la problématique des rapports entre «fragment », «forme brève » et « écriture discontinue » chez Barthes, voir Claude Coste (2009).
} 
réalise une figure d'auteur qui une fois mise en abyme, ne cesse de croître dans l'éclatement des formes iconographiques et sémantiques du Moi. Par conséquent, la rhétorique autoportraitiste et évasive de Barthes amplifie cette écriture fragmentaire par laquelle l'écrivain respire et vit.

\section{Atiq Rahimi et l'art des figures inachevées}

Alors qu'Édouard Levé réalise son autoportrait par une somme d'énoncés brefs, Roland Barthes s'étale en propos dubitatifs sur lui-même jusqu'à remettre en question le processus de l'autoreprésentation. D’autres laissent leur figure d'auteur en suspens, abandonnant la forme sur une traverse entre deux rives que sont l'unité et l'éclatement. Je terminerai donc avec ce dernier exemple de traitement formel qui endosse l'oxymore des figures inachevées. C'est dans La ballade du calame, un autoportrait à la fois littéraire et visuel, que le FrancoAfghan Atiq Rahimi transforme des calligraphies persanes en croquis, ceux-ci représentant, soit des figures féminines, soit des symboles mystiques témoignant de ses postures idéologiques ou de sa phénoménologie d'existence.

L'œuvre s'annonce sur la page couverture comme étant un « Portrait intime » par la voie duquel l'auteur reste tributaire d'un dédoublement au cœur de son autoreprésentation. Contrairement à l'usage de l'éclatement chez Barthes et Levé, le premier projetant le fragment vers des topiques extérieures et le deuxième le ramenant vers une intériorité subversive, Rahimi se rallie à l'écriture fragmentaire dans la déchirure d'une dualité qui se lit entre dessin graphique et poésie en prose. L’artiste invente cet alliage intermédial qu'il nommera, «callimorphies ». Le néologisme indique la base d'une calligraphie persane transmutée sous la plume du calame, soit en corps de femmes en tant que vecteurs de topiques autoportraitistes, soit en métaphore qui, d'un seul geste, illustre le texte accompagnateur sous 
formes de signes en mouvement. Quant aux femmes représentées, ce sont des corps qui se lovent à la page blanche en lignes continues, enroulées de galbes qui se dispersent et qui s'effacent, laissant la forme en état d'ouverture. Les callimorphies incarnent ainsi, des figures inachevées.

Voici quelques repères pour situer l'artiste qui en fait, est à la fois cinéaste, romancier, poète et photographe. Atiq Rahimi a fui l’Afghanistan dans les années 1980 pour se réfugier en France. Il obtient la nationalité française et termine ses études doctorales à la Sorbonne en audio-visuel. La traversée de ces multiples frontières l'a mené à vivre dans une langue étrangère en tant que réfugié, ainsi qu'à assimiler une culture fondée davantage sur l'image que sur les mots, lui qui venait d'un pays où le verbe et la poésie dominent la culture. Ses influences provenant de la littérature française et des écrits mystiques du Moyen-Orient, traduisent non seulement les vides et les pleins de l'exilé, mais elles mettent en exergue l'errance interne et externe du sujet.

Toutes ces expériences initiatiques forment le consort des épreuves qui ont consacré la facture artistique d'Atiq Rahimi. Son esthétisme en reste profondément imprégné, car chacune de ses créations reflète non seulement les impasses de sa double identité francoafghane, mais chacune établit des rapports entre les faits biographiques et des traitements formels variés qui toujours, tanguent entre les arts visuels et l'univers de l'écriture. C'est un être dualiste jusque dans sa doublure esthétique. Cette démarche artistique appelle donc à la négociation sans fin de ses propres doubles, si bien que la traversée des frontières esthétiques devient une subversion nécessaire à son art. Plus précisément, c'est dans l'entre-deux que Rahimi trouve ses réponses et que, à l'égal de Roland Barthes, il développe cet amour et ce désir de la forme incomplète : «En callimorphie, je ne sais achever un corps. Je l'inachève.» (Rahimi, 2015, p. 173) Les croquis relèvent davantage de l'exercice initiatique en s'éloignant 
nettement du dessin académique, et notamment, la frivolité des traits véhicule les affects et les percepts des faits biographiques précédemment racontés dans le texte.

Chaque fragment réalise une figure à partir d'une lettre calligraphique qui devient corps ou symbole, et à partir duquel un mouvement est perçu. Mais c'est surtout l'écriture accompagnant la callimorphie qui, en quelque sorte, l'enveloppe de sens. Le texte fragmentaire jumelé à l'image d'une forme anthropomorphique inachevée produit des figures d'auteur doubles : l'autoportrait résultant d'un dialogue entre deux médiums. Par exemple, la callimorphie intitulée «Errant» (Rahimi, 2015, p.182), qui en soi, constitue la chute de l'œuvre, est plus aérienne que la calligraphie à partir de laquelle l'auteur s'inspire (la comparaison est rendue possible grâce à la « Table des callimorphies » (p. 191) où se trouvent les calligraphies en écriture persane et d'où émerge le mouvement subversif du calame). La forme s'étire comme une trace de vent qu'un voyageur aurait laissée derrière lui. La pulsion des tracés s'élève vers le haut et se propulse au-devant. Le signe possède l'élan du fugitif, mais le trait crée une cassure dans son ombre tel un miroir fracturé. «Errant» devient alors la représentation parfaite de l'auteur, car ce dernier a transformé le signifiant du signe calligraphique afin de rapprocher son signifié de sa figure d'autoportrait. Notamment, Rahimi revient sans cesse sur les fêlures de son errance, tel un socle planté dans son être. 


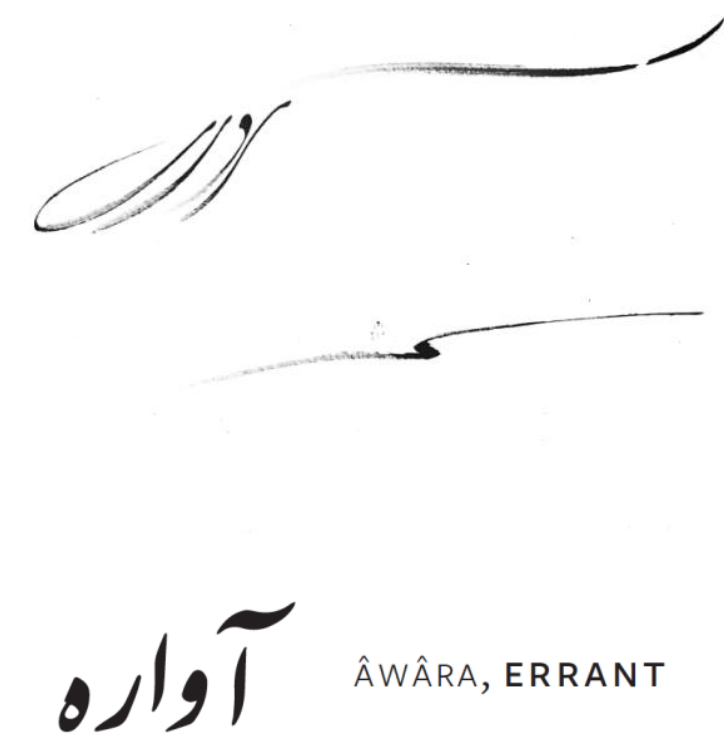

(C) Reproduction autorisée par Atiq Rahimi

Un deuxième exemple réconcilie l'auteur avec un certain sens d'unité entre deux opposés tant dans la forme qu'au niveau du contenu. Partant d'une lexie de la langue persane que représente le mot djân ${ }^{10}$, Rahimi combine rhétorique d'arguments avec rhétorique de figures. Le terme d'origine désigne le corps-âme sans dualisme. Contrairement aux notions de chair et d'esprit, qui sont généralement interprétées dans l'histoire de la pensée occidentale (platonicienne et judéo-chrétienne) comme étant des instances opposées, la langue perse leur attribue une seule et même idée. Le corps et l'âme ne sont aucunement séparés.

Indisposé à reproduire la calligraphie d'origine du djân, Atiq Rahimi lui restitue une image qui suggère un ventre engrossé par la vie d'un être invisible, mais dont la trace du parcours aurait laissé ses palimpsestes tout juste devant (p.137), comme les sillons d'un

\footnotetext{
10 Voir le poème « Les Djinns » de Victor Hugo (1829). Ne pas confondre djinn et djân, car le premier terme réfère à une créature surnaturelle, invisible ou protéiforme, capable d'influencer les humains.
} 
soupir assurant une continuité entre la vie et la mort. Cette lecture du dessin n'est qu'une interprétation qui pourrait bien être réfutée par l'auteur lui-même, mais le texte qui succède immédiatement cette callimorphie du corps-âme, semble être le reflet sensible du tracé frivole d'un errant ou encore, de l'exil qu'a vécu Rahimi et qui se poursuit en lui-même.

Lorsque je regarde mon image, une étrange sensation s'empare de moi. Je redeviens enfant; non, pas enfant, un être primitif. Celui qui découvre pour la première fois l'ombre de son corps, ou son reflet sur la surface de l'eau, bien avant la naissance du miroir... Oui, je suis cet homme sauvage qui, inquiet et émerveillé, interroge son image : «Qui es-tu?»

D'où surgit cette question?

De mon âme ou de mon corps?

D'aucun.

Je ne suis pas corps-et-âme.

Je ne suis pas seulement le corps, non plus.

Ni l'âme.

Ni le corps de l'âme.

Ni l'âme du corps...

Je suis djân. (Rahimi, 2015, p. 137-138)
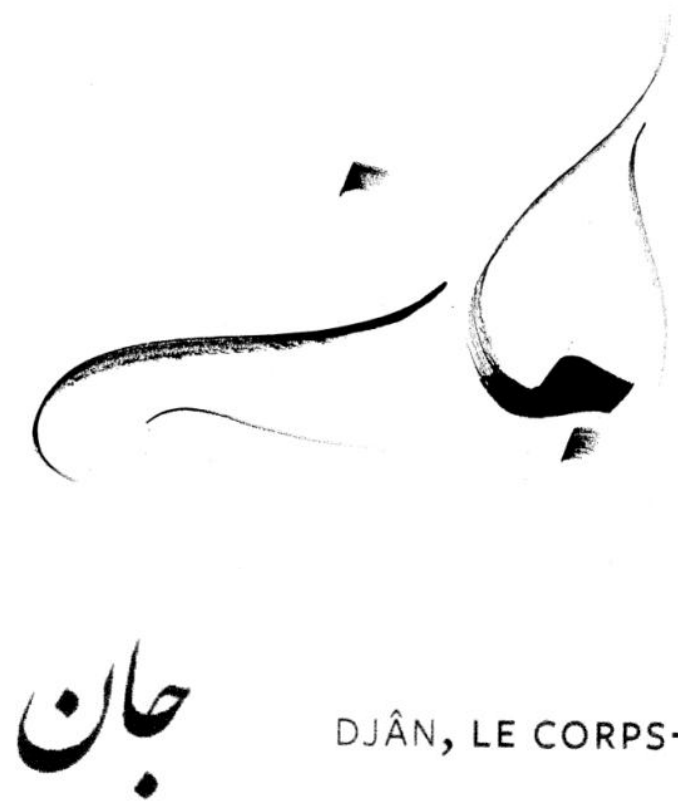

DJÂN, LE CORPS-ÂME

(C) Reproduction autorisée par Atiq Rahimi 
Le texte se clôt sur une figure d'autoportrait s'inspirant tout d'abord de la langue maternelle de l'auteur. Et de concert avec le sens du mot djân, le fragment réunit une multitude d'éléments en illustrant l'acte autoportraitiste en tant que tel. Tout d'abord, la question existentielle du Moi : «Qui es-tu?»; fait directement allusion au reflet de Narcisse dans Les métamorphoses d'Ovide (1992); l'un des premiers textes de l'Antiquité qui dépeint l'autoportraitisme par une rencontre solipsiste entre l'égo et son double avant même l'apparition du miroir ${ }^{11}$. Enfin, la suite du texte témoigne du caractère instinctif de la démarche artistique de Rahimi en définissant la posture dite "primitive » de son intériorité. " Je suis djân», s'avère ainsi une rhétorique de figure qui caractérise à la fois l'impasse et l'issue de l'écrivain. Or, dans toutes les callimorphies créées par l'auteur, l'oxymore des figures inachevées se résout par la rhétorique du fragment textuel, celle-ci s'inspirant du signe, qui une fois transformé par le calame d'Atiq Rahimi, parachève le sens de la topique. L'autoportrait demeure le produit d'une intermédialité riche en dialogues et d'où émerge une figure d'auteur ancrée dans une pratique d'écriture fragmentaire, brève et inachevable.

\section{Conclusion : l'autoportrait littéraire entre l'éclatement et l'unité de l'ouvre}

Les trois illustrations du corpus sont des exemples de traitements formels parmi tant d'autres. Parce que l'autoportrait parle d'un être particulier au parcours unique, l'enjeu de sa création en est un d'appropriation. L'acte autoportraitiste devra trouver sa manière de faire, se frayer une voie sémantique avec ses inventions particulières. J'ai choisi l'exemple de Levé afin de démontrer un système d'idiosyncrasies fragmentées en de courtes syntaxes, celles-ci étant

\footnotetext{
11 Voir Les métamorphoses d’Ovide (1992), p. 117-123. Sur les enjeux historiques du miroir, voir également Sabine Melchior-Bonnet (1994).
} 
parvenues à représenter une partie de la personne qu'il est. Pour son penchant contraire, j'ai cru bon de désigner Barthes, car l'auteur incarne la force de la pensée libre qui voyage à travers l'expérience du langage, ainsi qu'il insiste sur l'infinitude de l'être menant à l'impasse même d'une autoreprésentation totalisante. La somme d'une vie demeure pour lui, une incalculable impossibilité. Quant à Rahimi, c'est un tango intermédial entre une multitude de doubles dont sa double identité s'avère l'enjeu principal de son autoreprésentation. La fragmentation de l'être s'appuie sur les écarts et les rapprochements de ces doubles. Une déchirure émane des figures inachevées et de l'écriture fragmentaire d'Atiq Rahimi où chaque fragment résonne avec la topique-mère au double balancier se tenant au cœur du sujet. Cette topique récurrente pourrait se résumer principalement à la figure de l'errant en exil.

Dans Bagatelles pour l'éternité. L'art du bref en littérature, l'article de Jacques Poirier portant sur «L'écriture de soi par instantanés », résume quelques propos sur le fragment qui renvoient à ma question du début, à savoir en quoi le fragment s'avère-t-il une pratique d'écriture privilégiée dans la représentation d'un autoportrait :

En même temps que se déconstruisent les idéologies et que vacillent les grands systèmes, toute une famille d'écrivains renonce à produire le roman de leurs vies, préfère offrir au lecteur une série d'instantanés, de scènes fantasmatiques, et propose des autoportraits qui sont autant d'albums photographiques, ainsi qu'on le voit dans des œuvres comme Le Traité des saisons d'Hector Bianciotti, Viva la muerte de Fernando Arrabal, ou Roland Barthes par lui-même. [...] Et, nous le verrons, ce que le recours à la forme brève dans l'écriture de soi tente de reproduire, c'est un rapport privilégié au processus de l'anamnèse, à ce mode privilégié de la temporalité psychique qu'est l'instant et à la force du trauma. (p. 168)

Le fragment s'ouvre sur un terrain d'écriture qui concorde avec le caractère fragmenté de nos activités cognitives : mémoires, affects, réflexions, imagerie mentale, percepts, bribes de textes, de films ou de chansons, rêves brisés, cauchemars vécus, intellectualisation et sentimentalisme. Ce sont tous des éléments qui contribuent à une phénoménologie d'existence, c'est-à-dire à un senti et à un perçu relevant d'un individu, seul tributaire de son 
parcours. L'écriture fragmentaire offre ainsi une panoplie de représentations versatiles qui permettront la saisie de tous les interstices visibles et invisibles du sujet. À l'énoncé unique et complexe que comporte la personne de l'écrivain, le fragment répond de mille énonciations entre l'éclatement des figures et l'unité de l'œuvre.

\section{Bibliographie}

Académie des lettres du Québec, fiche technique de Nicole Brossard, http://www.academiedeslettresduquebec.ca/membres/nicole-brossard-39.

BARTHES, Roland (1957), Mythologies, Paris, Seuil.

BARTHES, Roland (1972 [1953]), Le degré zéro de l'écriture, Paris, Seuil.

BARTHES, Roland (1975), Roland Barthes par Roland Barthes, Paris, Seuil.

BARTHES, Roland (2009), Journal de deuil, Paris, Seuil/Imec, coll. « Fiction et Cie ».

BeAujour, Michel (1980), Miroirs d'encre. Rhétorique de l'autoportrait, Paris, Seuil.

BLANCHOT, Maurice (1988 [1955]) L'espace littéraire, Paris, Gallimard, coll. « Blanche ».

Blanchot, Maurice (1969), L'entretien infini, Paris, Gallimard, coll. « Blanche ».

BlAnCHOT, Maurice (1973), Le pas au-delà, Paris, Gallimard, coll. « Blanche ».

Blanchot, Maurice (1980), L'écriture du désastre, Paris, Gallimard, coll. « Blanche ».

Coste, Claude (2009), «Roland Barthes par Roland Barthes ou Le démon de la totalité », Recherches et travaux, no 75, p. 35-54, http://journals.openedition.org/recherchestravaux/372.

GUIBERT, Hervé (1981), L'image fantôme, Paris, Minuit.

Hugo, Victor (1829), Les orientales, Paris, C. Gosselin/H. Bossange.

JAKOBSON, Roman (1963), Essais de linguistique générale, Paris, Minuit.

LEJEUNE, Philippe (1975), Le pacte autobiographique, Paris, Seuil.

LEVÉ, Édouard (2008), Suicide, Paris, P.O.L. 
LEVÉ, Édouard (2013 [2005]), Autoportrait, Paris, P.O.L.

LEVÉ, Édouard (2013 [2005]), Autoportrait, dessin pointilliste ayant servi de page couverture, http://urlz.fr/6jk2.

MELCHIOR-Bonnet, Sabine (1994), Histoire du miroir, Paris, Hachette Littératures, coll. «Pluriel».

OviDE (1992), Les métamorphoses, édition de Jean-Pierre Néraudau, Paris, Gallimard, coll. « Folio classique».

POIRIER, Jacques (2000), «L'écriture de soi par instantanés », dans Philippe Baron et Anne Mantero (dir.), Bagatelles pour l'éternité. L'art du bref en littérature, Besançon, Presses universitaires franc-comtoises, p. 167-178.

RAHIMI, Atiq (2015), La ballade du calame, Paris, L'Iconoclaste.

Susini-AnASTOPoulos, Françoise (1997), L'écriture fragmentaire. Définitions et enjeux, Paris, Presses universitaires de France, coll. « Écriture ».

WATT, Adam (2014), « Reading Proust in Barthes's Journal de deuil », Nottingham French Studies, vol. 53, no 1, p. 102-112, http://www.euppublishing.com/doi/abs/10.3366/nfs.2014.0076.

WiLDE, Oscar (1997), Aphorismes, traduction de Bernard Hoepffner, Paris, Mille et une Nuits.

\title{
Résumé
}

Cet article veut rendre compte des différents traitements qu'emprunte le texte fragmentaire au sein de l'autoportrait littéraire. Après une courte introduction théorique sur le fragment en tant que pratique d'écriture, je le présente d'abord comme étant un traitement formel capable d'atteindre à l'unité d'une œuvre avec Autoportrait, d'Édouard Levé (2013 [2005]). Ensuite, il sera question de l'impossible totalité dans l'autoreprésentation avec Roland Barthes par Roland Barthes (1975). Et pour terminer, j'aborde la part que joue la prose poétique en compagnie des figures inachevées d'Atiq Rahimi dans La ballade du calame (2015). Les figures de Levé et de Rahimi se consolident davantage dans une topique-mère que dans l'autoportrait de Barthes qui quant à lui, se déconstruit par le biais d'une écriture fragmentaire encore voisine de la désarticulation.

\begin{abstract}
This article presents the different forms and styles that fragmentary writing could bring to a literary self-portrait. After a short theoretical introduction on the fragment as a writing practice, I first present an aesthetic form that is based on the sense of unity within a fragmented piece of work with, Autoportrait, from Édouard Levé (2013 [2005]). Then, I will debate on the impossible wholeness in the self-portraying of Roland Barthes par Roland
\end{abstract}


Barthes (1975). At last, I will analyse the part being played by the prose poem with Atiq Rahimi and his unfinished figures in La ballade du calame (2015). Levé's and Rahimi's fragments are more likely to deploy their multitudes under a unifying theme. However, Barthes's work, instead of building up around a topical center, deconstructs with a typical fragmentary writing that remains very close to dismemberment. 